\title{
Empirical Exploration of the time interval distribution of terrorism events in several Asia countries
}

\author{
Junfang Zhu' ${ }^{1,}$, Beilei Tong ${ }^{1, b}$, Peijie Ma ${ }^{1, b}$ \\ ${ }^{1}$ School of Science, Southwest University of Science and Technology, Mianyang, 621010, Sichuan, \\ China \\ aemail: zjfbird@swust.edu.cn, ${ }^{\text {bemail: tongbeilei@swust.edu.cn, }{ }^{c} e m a i l: m p j \_1982 @ s i n a . c o m ~}$
}

Keywords: Terrorism; Statistic analysis; Burstiness; Memory

\begin{abstract}
In recent years, people have found the power law distribution appears in natural and human activities, even in the event of terrorism. However, In this paper we analyze the time interval distribution of terrorism events in several Asia countries, and find that not all of the time interval distributions are showing a power law distribution. The time interval distribution of terrorism events in China is exponential distribution. Through calculating burstiness parameter and memory parameter it is found that power-law distribution is origin from strong burst and the burst and memory are relatively weak corresponding to and the time interval exponent distribution of terrorism events in China.
\end{abstract}

\section{Introduction}

It is confirmed by a large number of facts that power law distribution appears in human activities $[1,2,3,4]$. Terrorism activities is a kind of destructive human activity. Due to complexity and secrecy, it is difficult to directly detect the internal operation mechanism, But we can known the pattern of the events and detect the further causes of the pattern. At present, the description of terrorist incidents has been publicly available in some databases. It has been concerned to detect the law of terrorist incidents from these databases $[4,5,6,7]$. In this paper, the time interval distribution of the terrorist events is extracted from the database and the causes is explained.

\section{The source of data}

All the data is from the Global Terrorism Database (GTD) (www.start.umd.edu/gtd) in this paper. It is an open-source database including information on terrorist events around the world from 1970 through 2014. Unlike many other event databases, the GTD includes systematic data on domestic as well as international terrorist incidents and now includes more than 140,000 cases. The form of original data is shown in Fig.1. It includes ID, date, country, city, perpetrator group fatalities, injured and target type. From the database we can know a brief description about events and the political background. So in order to obtain the specific quantitative statistics distribution, we have to deal with the data to describe the statistic feature by methods of statistical physics. 


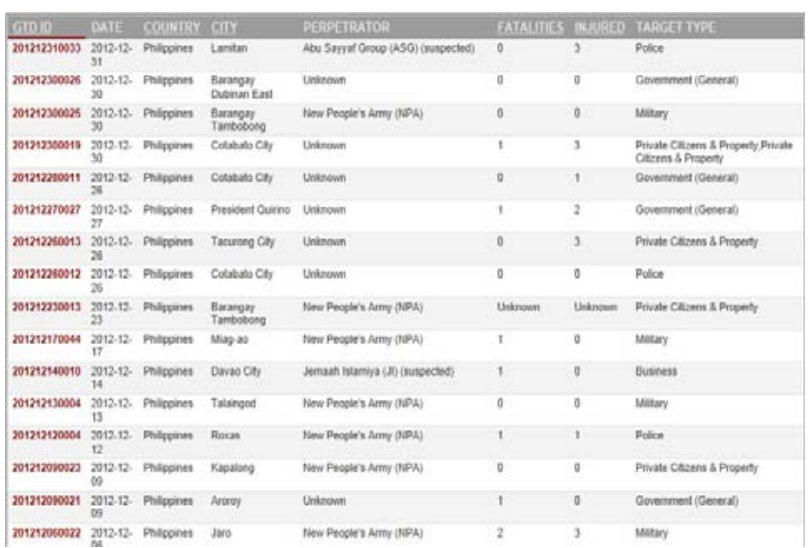

Fig.1. the source of data

\section{Statistic analysis}

We get the time interval according to the date of the event, and then the time interval between two consecutive events is ranked to get the time interval statistic feature.

Terrorism events are studied in Turkey, Lebanon, Philippines and China over the last forty years. In order to quantitatively describe the events properties, the temporal patterns as obtained in Fig. 2. It can be seen the events occurred frequently in some time and rarely in some other time. Obviously it means that the heterogeneous and uncertainty. However the occurrence of terrorism events seems to be relatively more homogeneous for China.

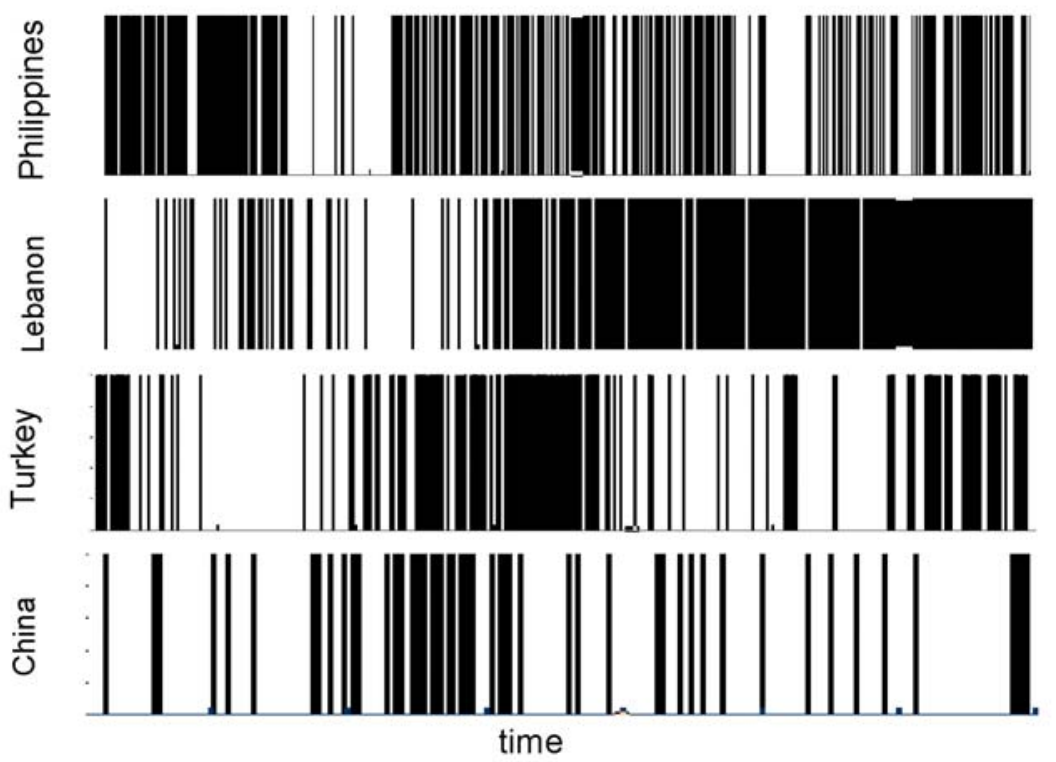

Fig.2. Series figure of events. The horizontal axis represents time and every line indicates an event.

In order to further clear its statistic feature, we obtained the Zipf's plot by ranked the time interval sequence of terrorism events in Fig. 3. we find that the ranked time interval are almost a line in the $\log$-log coordination for Philippines, Lebanon and Turkey which shows that the time interval distribution is like power law distribution In figure 3. If the power law exponent is $\alpha_{z}$ in Zipf's plot, then the time interval follows the probability distribution $p(\tau)=\tau^{-\alpha}$ with exponent $\alpha$ satisfying the formula $R^{-1 / \alpha_{z}}=\int_{R}^{\infty} p(\tau) d \tau \propto R^{-(\alpha-1)}$ [8,9]. Here $\mathrm{R}$ represents the rank. According to the above formula, we can get $\alpha=1+1 / \alpha_{z}$. Therefore, time interval distribution has power exponent $\alpha=2.37, \alpha=2.56, \alpha=2.40$ respectively for Philippines, Lebanon and Turkey. However 
it is in the normal-log coordination the fitting is a straight line. Obviously the time interval distribution is according with exponent distribution.
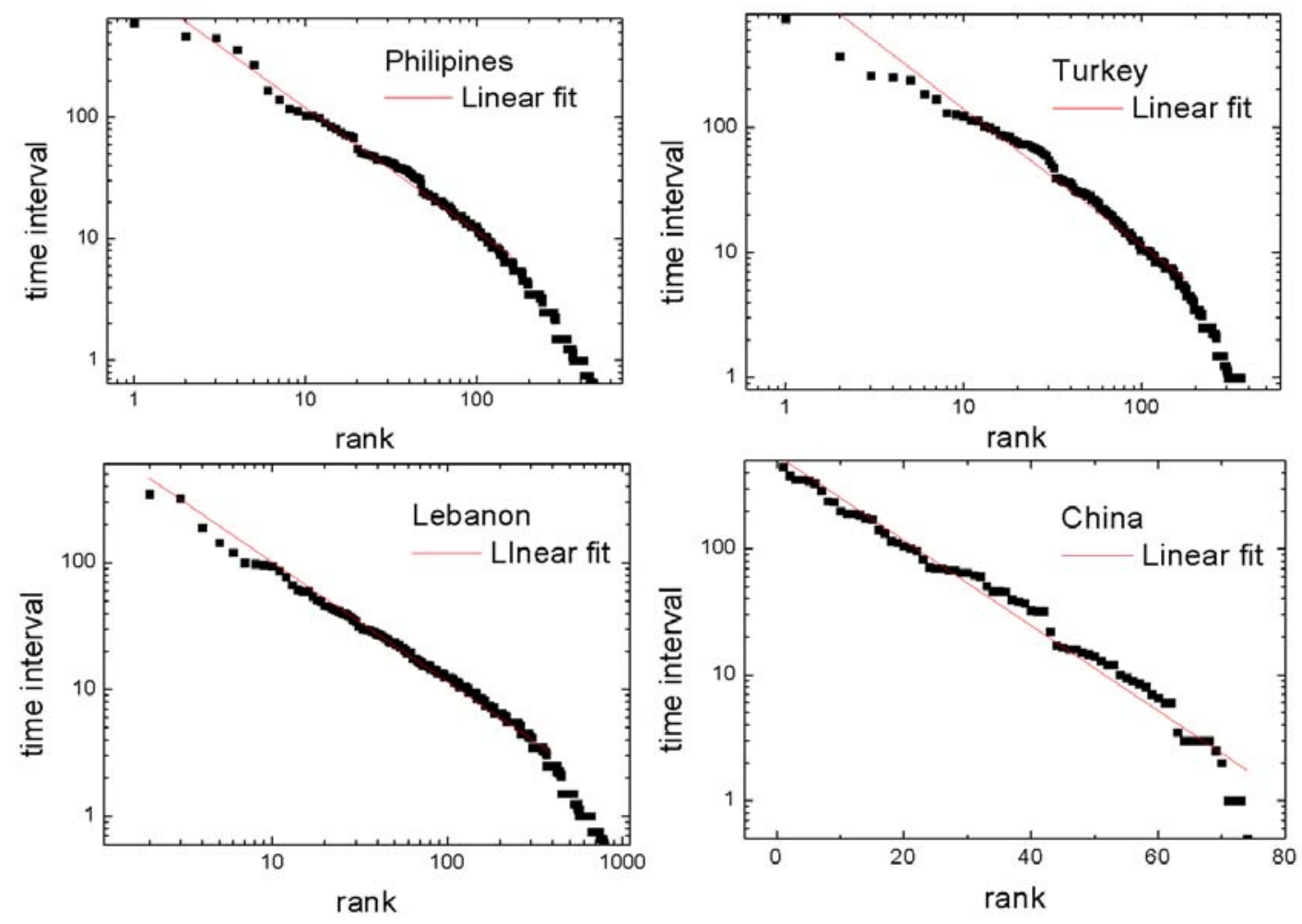

Figure.3 The Zipf's plot of terrorism events

\section{Explanation}

The above empirical analysis shown the time interval distribution of terrorism events in China is different from other Asian countries. Why there are so much differences of the statistic distribution? Many papers have illustrated that the importance of $[10,11,12]$ bursty and memory for statistical law. Therefore, in order to find out the reasons, we calculate the burst parameter $B$ and memory coefficient $M$ for time interval of terrorism events.

$$
\begin{aligned}
& B=\frac{\sigma_{\tau}-m_{\tau}}{\sigma_{\tau}+m_{\tau}} \\
& M=\frac{1}{n_{\tau}-1} \sum_{i=1}^{n_{\tau}-1} \frac{\left(\tau_{i}-m_{1}\right)\left(\tau_{i+1}-m_{2}\right)}{\sigma_{1} \sigma_{2}}
\end{aligned}
$$

Here, $\sigma_{\tau}$ represents the standard deviation and $m_{\tau}$ is the mean value. The value of two parameters are both in the range $(-1,+1)$. For burstiness parameter, $B=1$ is the bustiest signal. $B=0$ is neutral and $B=-1$ indicates the regular signal. Memory parameter is a correlation-based measure. it is positive when the short (long) time interval tends to be followed the short (long) time interval, it is negative if the short (long) time interval is followed by long (short). After calculation, we obtain the values of burstiness parameter and memory coefficient shown as Tab.1. The result proves the strong burst and memory for the time interval in Philippines, Lebanon and Turkey which is why time interval distribution presents like power law distribution. The burst and memory is lack for time interval of terrorism event in China, so randomness of events is relatively larger.

Tab.1. Burstiness parameter and memory coefficient

\begin{tabular}{|c|c|c|c|c|}
\hline & Philippines & Lebanon & Turkey & China \\
\hline Burstiness prameter & 0.587 & 0.587 & 0.555 & 0.126 \\
\hline Memory coefficient & 0.331 & 0.59 & 0.303 & 0.27 \\
\hline
\end{tabular}




\section{Conclusion}

The occurrence time of terrorism events is acquired from the open database GTD in four countries in Asia. We extracted and analysis the time interval. Moreover, we found the time interval distribution follows like power law for Philippines, Turkey, Lebanon and exponent distribution for China. Further exploration shows strong burst and memory is the origin of like power law distribution and exponent distribution has the weak burst and memory.

\section{Acknowledgement}

This research was financially supported by key Fund Project of Sichuan Provincial Department of Education (Grant No.13ZA0168) and the school Fund of Southwest University of science and technology (Grant No.10zx7138).

\section{References}

[1] Jean Pierre Eckmann, Elisha Moses and Danilo Sergi Entropy of dialogues creates coherent structure in email traffic, Proc. Natl. Acad. Sci. U.S.A. 2004, 101, 14333-14337

[2] Wei Hong, Xiao Pu Han, Tao Zhou and Bing Hong Wang, Scaling behaviors in Short-message communications, Chin. Phys. Lett. 2009, 26, 028902

[3] Alexei Vázquez, Joäo Gama Oliveira, Zoltán. Dezsö, Kwang-II Goh, Imre Kondor And Albert-László Barabási, Modeling burst and heavy tails in human dynamics, Phys. Rev. E., 2006, 73, 036127.

[4] Fa Dong Zhao, Hong Wei Zhuang and Zhen Xin Jin, Empirical studies on attack patterns of terrorism groups based on MLE. Complex System and Complexity Science, 2014,11, 4, 19-22.

[5] A. R.Jose R.Eduardo and U.Rafae, Scale omvariance om the 2003-2005 Iraq conflict, Physica A, 2007, 337, 291-301

[6] Jun Fang Zhu, Bing Hong Wang, Statistical law of moern terrorism, Journal of University of Shanghai for Science and Technology, 2012, 34, 3, 240-251.

[7] Telesca Luciano and Lovallo Michele Are global terrorist attacks time-correlated? Physica A: Statistical Mechanics and its Applications, 2006, 362, 2, 480-484

[8] George Kingsley Zipf, Human Behavior and the Principle of Least Effort: An Introduction to Human Ecology (Cambridge, MA: Addison-Wesley) 1949

[9] M. E. J. Newman, Contemporary Physics, 2005, 46, 323

[10] K. I. Goh and A. L. Barabasi, Burstiness and memory in complex system, EPL, 2008, 81, 48002.

[11] Shi Min Cai, Zhong Qian Fu, Tao Zhou, Jun Gu And Pei Ling Zhou, Scaling and memory in recurrence intervals of internet traffic, EPL, 2009, 87, 69001

[12] Valerie N. Livina, Shlomo Havilin and Armin Bunde, Memory in the Occurrence of Earthquakes, Phys. Rev. Lett. 2005, 95, 208501 